\title{
LA RESILIENCIA EN LOS CONTEXTOS DE REFUGIO REVISIÓN BIBLIOGRÁFICA
}

\section{THE RESILIENCY IN REFUGEE CONTEXT. A REVIEW ARTICLE.}

\author{
Iciar Villacieros Durbán
}

Universidad Pontificia de Comillas, Madrid, España.

Cómo referenciar este artículo/How to reference this article:

Villacieros Durbán, I. (2016). La Resiliencia en los contextos de Refugio. Revisión bibliográfica. Revista de Psicoterapia, 27(105), 139-155.

\begin{abstract}
Resumen
Desde el año 1955, el estudio de la resiliencia, ha ido creciendo en diversas áreas del conocimiento (Santos da Silva, Lacharite, Arruda da Silva, Lerch y Lundardi, 2009). Desde los primeros estudios longitudinales que se realizaron (Werner y Smith, 1982), hasta la actualidad, se ha tratado de un fenómeno complejo, que asume una mayor importancia en contextos de riesgo (Santos da Silva, et al., 2009). De hecho, son los estudios de poblaciones rurales en extrema pobreza, los primeros en demostrar la existencia de este concepto y sus implicaciones a nivel familiar (Werner y Smith, 1982). De igual modo, el concepto de resiliencia es un acercamiento con grandes ventajas para acercarnos al fenómeno de las migraciones, especialmente cuando pobreza y migración se solapan y comparten sus principales características (Guribye, 2011). Definimos resiliencia como: "La capacidad de la persona para recobrarse de la adversidad fortalecida y dueña de mayores recursos, Walsh (2004, p.26)". Esta capacidad ha sido estudiada habitualmente desde la mirada del traumatismo individual, sin observarlo en su contexto y de forma dinámica. En cambio, el concepto de resiliencia familiar, pondría el foco en superar la crisis y el desafio desde la herramientas del propio sistema familiar movilizando ciertos recursos para hacerle frente, a pesar de parecer superadas por la situación (Delage, 2010). Tal y como explica Benzies y Mychasiuk (2009) la resiliencia familiar no se desarrolla por medio de la evasión, sino poniendo en marcha mecanismos protectores para enfrentar las situaciones de adversidad y salir de ellas más fortalecido. En esta revisión bibliográfica de las investigaciones más actuales en resiliencia y refugio encontramos algunos de los factores las claves. Además, se propone el modelo ABCX (McCubbin, 1983) como un acercamiento aplicable al fenómeno de la resiliencia en contexto de refugio desde su dimensión de la familia. Por último se exponen algunas conclusiones y futuras investigaciones sobre el tema.

Palabras clave: resiliencia, refugio, trauma, familia.
\end{abstract}

Fecha de recepción: 18/07/2016. Fecha de aceptación v1: 13/09/2016. Fecha de aceptación v2: 7/10/2016 Correspondencia sobre este artículo:

E-mail: ivillacieros@gmail.com

Dirección postal: Universidad Pontificia de Comillas, Madrid, España.

(C) 2016 Revista de Psicoterapia 


\section{Abstract}

Since 1955, resiliency studies have been published in different disciplines (Santos da Silva, Lacharite, Arruda da Silva, Lerch, \& Lundardi Filho, 2009). The first longitudinal studies (Werner \& Smith, 1982) show the complexity of resiliency, and how it increases in risk contexts (Santos da Silva and cols, 2009). In fact, researches performed in the context of extreme rural poverty where the first ones in validating the concept of resiliency and its implications for families (Werner \& Smith, 1982). Thus, resiliency can be an appropriate approach to the study of international migrations, especially in conjunction with poverty (Guribye, 2011). Resilience is defined as "people's capacity of recover from adversity, and get stronger and with more resources Walsh (2004, p.26)". This capacity has usually been studied considering the different elements of individual trauma, leaving aside its contexts and dynamic aspects. However, the familiar resiliency approach puts the spotlight on overcoming the crisis using the familiar system tools, their resources too face it, even when they seem overwhelmed (Delage, 2010). As shown by Benzies y Mychasiuk (2009), familiar resilience does not appear when avoiding adverse situations; but while facing these situations. The results of this process are strengthened people and families. In this research performed over the most current investigations run on resilience and shelter, we can find some of the key drivers of the key elements. Also, the ABCX model (McCubbin, 1983) is proposed as an approach applicable to the resilience phenomenon in the refuge from its family dimension context. Closing the document presents some conclusions and future researches in progress on the subject.

Keywords: resiliency, refugee context, trauma, family. 


\section{Introducción: El refugio como situación traumática.}

La Convención sobre el Estatuto de los Refugiados (Ginebra 1951) y su Protocolo de Nueva York en 1967, en el Artículo 1A (2) define a un refugiado como cualquier persona que: "Debido a fundados temores de ser perseguida por motivos de raza, religión, nacionalidad, pertenencia a un determinado grupo social u opiniones políticas, se encuentre fuera del país de su nacionalidad y no pueda o, a causa de dichos temores, no quiera acogerse a la protección de al país; o que, careciendo de nacionalidad y hallándose, a consecuencia de tales acontecimientos, fuera del país donde antes tuviera su residencia habitual, no pueda o, a causa de dichos temores, no quiera regresar a él “ (Acnur, 2016). Por lo tanto necesita acogerse a la protección internacional.

En el año 2014 el Alto Comisionado para las Naciones Unidad (ACNUR) registró 866.000 nuevas solicitudes de asilo en el mundo, 269.400 más que el año anterior. Se calculan que son los segundos niveles más altos a nivel mundial desde los años 80. Entre los principales países receptores, Europa recibió un total de 714.300 solicitudes, que suponen el $80 \%$ de todas las nuevas solicitudes presentadas en el año. La mayoría personas que cruzaron el mediterráneo procedentes de la República Árabe Siria y Eritrea. En Norteamérica se presentaron 134.600 solicitudes, donde más de la mitad procedían de México (Acnur, 2014).

El Informe Tendencias Globales 2015 del Alto Comisionado de Naciones Unidas para los Refugiados (ACNUR) afirma que el desplazamiento forzado global ha aumentado en 2015, alcanzando una vez más niveles sin precedentes. Al término del año, había 65,3 millones de personas desplazadas forzosamente en todo el mundo a consecuencia de la persecución, los conflictos, la violencia generalizada o las violaciones de derechos humanos. Esto son 5,8 millones de personas más que el año anterior - 59,5 millones- (Acnur, 2015). La población global sometida a desplazamiento forzado se ha incrementado de manera sustancial durante los últimos dos decenios, pasando de 37,3 millones en 1996 a 65,3 millones en 2015: un aumento del $75 \%$. Desde 1999 hasta 2011 esta población permaneció relativamente estable, fluctuando en torno a unas seis personas por cada 1.000 de la población mundial o una de cada 167 personas. No obstante, desde entonces, y coincidiendo con el comienzo de la "Primavera Árabe" y del conflicto sirio, esta proporción se ha incrementado de forma acusada y al concluir 2015 era de casi nueve de cada 1.000 personas o una de cada 113 personas (Acnur, 2015).

El conflicto en la República Árabe Siria, que entró en su quinto año en 2015, recibió una atención mundial significativa debido a las grandes entradas de refugiados y a las necesidades humanitarias generadas. No obstante, contribuyeron también al aumento de la población mundial en situación de desplazamiento forzado otras crisis y conflictos no resueltos, así como los que surgieron a lo largo del año, como los nuevos conflictos de Burundi, Irak, Libia, Níger y Nigeria, y la reactivación de las crisis no resueltas de Afganistán, la República Centroafricana, la República Democrática del Congo, Sudán del Sur y Yemen (Acnur, 2015; CEAR, 2015). 
En algunos estudios se distingue el estatuto de refugiado de la figura del solicitante de asilo, en proceso de ser reconocida su condición de refugiado, como distintas figuras a nivel legal. Otras categorías son los desplazados internos, apátridas o los grupos de retornados. Este último grupo lo constituyen los que han sido reasentados en un país seguro. Por lo que se encuentran también estudios con esta población, realizados en el país de acogida (Boucher, 2009; Lewis, 2010; Shteir, 2009).

Aquellas personas que a pesar de vivir en contextos traumáticos como el refugio, desarrollan mecanismos sanadores, para ellos y sus familias saliendo fortalecidos son el objeto de esta revisión bibliográfica. Dado que las situaciones de violencia y trauma son una realidad continúa, el profundizar en esos mecanismos de resiliencia puede dar pie a futuros trabajos de prevención en contextos de trauma.

\subsection{Trauma y refugio:}

El contexto de refugio, conlleva numerosos traumas; el desplazamiento, las separaciones, la exposición a la violencia, la persecución o el maltrato son algunos de los eventos dolorosos que encontramos en la literatura (Hobfoll, 2011). Constituyen pérdidas tanto materiales, como emocionales o psicológicas (Horn, 2009). Por ejemplo, una de las más significativas es la pérdida del hogar. Para los niños estudiados por Rosbrook (2010) fue considerada como un evento traumático y estresante principalmente en aquellos que asociaban el hogar con la variable lugar seguro.

Desde la psicología clásica y la psiquiatría, se ha abordado la experiencia traumática mediante la medición de síntomas patológicos. En concreto midiendo depresión y trastorno de estrés postraumático (TEPT) según los criterios del Manual Diagnóstico y Estadístico de los Trastornos Mentales (DSM) en su quinta edición (Asociación Americana de Psiquiatría, 2013). Así se puede observar que, derivado de estas experiencias traumáticas, muchas personas desarrollan malestar emocional y sintomatología psiquiátrica. Hobfoll (2011) examina los límites de la resiliencia en una muestra de 1200 personas supervivientes de la violencia en Palestina, basándose en instrumentos clínicos encuentran una alta prevalencia de TEPT y depresión. En este estudio no se encuentran factores de protección ni desarrollo de resiliencia en estas mismas personas. En la misma línea, Vaage (2011) evalúa sintomatología psiquiátrica (TEPT y síntomas de ansiedad, depresión y agresividad) en la población de refugiados vietnamitas asentados en Noruega. Como resultado encontraron que el $30 \%$ de las familias tenía un progenitor con sintomatología psiquiátrica. Klasen (2010) examina una muestra de 330 adolescentes y niños soldados con edades comprendidas entre 11 y 17 expuestos a la violencia en Uganda, de los cuales solamente el 27,6\% no mostraron sintomatología de TEPT, de depresión, ni problemas conductuales o emocionales.

Sin embargo, también se puede observar la experiencia traumática desde el punto de vista de la resiliencia, o del desarrollo de mecanismos sanadores. 
La palabra resiliencia viene del término latino "resilio" que significa "volver atrás", o "volver de un salto" al estado inicial y fue utilizada originariamente para referirse a la cualidad de elasticidad de un material. El término fue adaptado a las ciencias sociales, para referirse a "Aquellas personas que a pesar de nacer y vivir en situaciones de alto riesgo se desarrollan psicológicamente sanos y exitosos" (Rutter, 1993).

Walsh (2004) define resiliencia como "La capacidad de la persona para recobrarse de la adversidad fortalecida y dueña de mayores recursos". Manciaux (2003) como "La capacidad de una persona o de un grupo para desarrollarse bien, para seguir proyectándose en el futuro a pesar de los acontecimientos desestabilizadores, de condiciones de vida difíciles y de traumas a veces graves".

Aunque no existe un consenso sobre la definición de resiliencia, en el presente artículo, nos referiremos a este último enfoque, desde donde hemos llevado a cabo la revisión bibliográfica.

\section{Factores de resiliencia en el contexto de refugio:}

Como se revisa a continuación, existen numerosos estudios sobre factores de riesgo y protección en el desarrollo de la resiliencia. En su mayoría, señalan que la ausencia de un factor protector, se convierte en uno de riesgo. Se presenta ahora un modelo que, aunque no fue creado para el fenómeno de la resiliencia, nos ayuda a enmarcar dichos factores de forma cronológica: antes, durante y después del trauma.

\subsection{El modelo de doble $\mathrm{ABCX}$ de adaptación familiar}

El modelo ABCX de adaptación familiar de McCubin y Patterson (1983) es una adaptación del modelo de Hill (1949), propuesto al final de la segunda guerra mundial, después de observar cómo afrontaban la separación y posterior reunión las familias en contextos estresantes.

Este modelo (McCubin y Patterson, 1983) no pretende explicar la sintomatología, sino que relaciona los factores que inciden en las familias y las respuestas, adaptativas o no. La propuesta se centra en las variables post trauma que la familia pone en juego para superar el estrés y adaptarse a la situación de la forma más exitosa posible. Además, sitúa la adaptación familiar inicial como mediadora entre el riesgo previo del menor y su adaptación psicosocial posterior.

Los componentes del modelo son: El factor estresante y las demandas acumuladas (factor A), los recursos familiares (factor B), la percepción de la familia sobre el evento estresante (factor $\mathrm{C}$ ) y el resultado de la adaptación familiar y del niño (factor $\mathrm{X}$ ).

El orden temporal de los factores A, B y C, resulta finalmente alterado, ya que las percepciones y evaluación que hace la familia, son una variable que afecta directamente a los recursos familiares que pone en marcha. Por lo tanto se trata más de un modelo ACB que ABC. 
Este modelo distingue dos momentos: la etapa de crisis, o fase de ajuste, en donde la familia pone en marcha solamente algunos mecanismos para afrontar estresores puntuales, y la etapa post crisis, o de adaptación, donde tiene que hacer una cambio estructural que garantice el equilibrio del sistema.

\subsubsection{EI factor A:}

Este factor se refiere por un lado a las características de evento estresante o traumático y por otro a la acumulación de estresores que conlleva. En el modelo doble se propone no sólo un factor puntual traumático, sino una acumulación de varias demandas que afectan al sistema familiar, aunque exista un elemento estresor que precipite la crisis. El afrontamiento no solo depende de este evento traumático sino de la configuración de estresores, demandas o tareas que se presentan a la persona.

\section{Características del trauma:}

Las características del trauma pueden influir en la resolución del mismo. Tomkiewitz (2004) afirma que la resiliencia tiene unos límites en la intensidad de su desarrollo que variarán en función de algunos aspectos; entre ellos de la cantidad de agresión y la calidad de agresión (puntual e intenso o crónico y prolongado).

La primera característica referida a la cantidad de agresión y su carácter persistente. Si los hechos estresantes se acumulan de manera continua como es el caso de los contextos de refugio y requieren un despliegue de recursos constante y demandante, el impacto será mayor (Achotegui, 2009). Respecto a la intensidad del trauma, ésta se ha estudiado en conflictos bélicos, como el palestino, donde los años de exposición lo convierten en un evento crónico. Los síntomas del TEPT y depresión son mayores cuando el evento traumático es prolongado y los recursos de resiliencia se ven sobrecargados (Hobfoll, 2011). En este mismo estudio, las personas que afirmaron estar menos expuestos a la violencia política, no haberla vivido directamente, ni haberla visto, desarrollaron menos síntomas de TEPT y depresión (Hobfoll, 2011), lo que señala la influencia de la menor intensidad de la vivencia traumática en su sintomatología.

\section{Acumulación de demandas:}

La acumulación de demandas estresantes se estudian en Bonanno (2010). Este autor explica la dificultad de encontrar resiliencia cuando el trauma es crónico ya que al darse una mayor acumulación de demandas es posible que los recursos estén sobrecargados y que los procesos de desarrollo de resiliencia sean más lentos. Así mismo, el trauma repetido, sumado a otros eventos estresantes como las condiciones de pobreza extrema y bajos recursos que padecieron los Palestinos encuestados dificulta la resiliencia (Hobfoll, 2011). Además, el hecho de iniciar el afrontamiento del evento estresante con niveles de sufrimiento mayores, conlleva un peor pronóstico ante el trauma (Hobfoll, 2011). 


\subsubsection{Factor C: Creencias, evaluación de la situación:}

$\mathrm{El}$ factor $\mathrm{C}$, se refiere a la evaluación y percepción que se hace de los eventos estresantes y de la demanda (Factor A). Según como sea esta evaluación, puede convertirse en factor de riesgo o protección para el desarrollo de mecanismos sanadores. De ahí su importancia como variable mediadora para la intervención terapéutica.

McCubbin (1983) lo define como "Percepción y coherencia" y supone la aceptación y entendimiento familiar para enfrentarse a la situación, en tres aspectos: control, sentido o "meaningfullness" y comprensión. El "control" se refiere al grado en que la familia cree que tiene de capacidades para afrontar el evento estresante. El "sentido" supone que a la familia le merece la pena enfrentarse al evento, o el significado que le dan al mismo. Y la "comprensión" alude a si el evento les resultaba esperable. Así, la percepción y la coherencia se convierten factores de mediación entre la crisis y la adaptación familiar.

Por lo tanto, en un primer nivel se encuentra lo predecible o expectativas inmediatas con respecto al trauma, en el que entrarían evaluaciones sobre si era esperado; Tomkiewitz (2004) afirma que una de las variables de las que depende la resiliencia es la imprevisibilidad o previsibilidad de la situación traumática. También Parkes (1988) apoya este mismo punto, añadiendo que los sucesos más peligrosos para la integridad psíquica son, entre otros, los que sobrevienen bruscamente sin tiempo de preparación.

En un segundo nivel estaría el sentido que se le otorga al trauma, refiriéndose a la tendencia a percibir el mundo de manera global como coherente y con sentido. Este concepto fue acuñado por Aaron Antonovsky en 1987, que afirma que el estado normal del ser humano en su medio ambiente es el desorden y el caos, y no la estabilidad. Por esta razón buscar conocer cuáles son los factores que promueven la estabilidad y encuentra el "sentido de coherencia" que lo define como una orientación global que ve la vida como algo razonable, manejable y significativo. Implica la confianza en que hay un sentido para las cosas que parecen desordenados, y que este sentido puede ser comprendido, previsible y controlado. Especialmente se ha desarrollado este concepto en los contextos de refugio a nivel comunitario, donde un mayor sentido de coherencia sobre los hechos ocurridos permitía predecir una mejor superación y adaptación, con resultados más satisfactorios en la comunidad. El uso de las historias, narrativas compartidas que dan un sentido a la crisis, como el método de "Línea del tiempo colectiva", que también se presunta un recurso protector para la muestra de refugiados en Rochester (Boucher, 2009).

Walsh (2004) habla de este segundo nivel, como "dar significado" o "make meaning" (meaningfulness) y lo define como: "Generar narrativas que den sentido a nuestras vidas, entre los cuales incluye tres aspectos: Dotar de sentido a la adversidad, perspectiva positiva y trascendencia y espiritualidad. Es el mismo término que Barudy (2006) describe como la importancia del "segundo golpe" o narración mental que se hacen de los hechos. Este autor estudia cómo la evaluación 
realizada por el propio contexto y familia da lugar a diferentes secuelas (TEPT) y/ o a un sentido individual y familiar. En el estudio de Boucher (2009) con refugiados asentados en Rorchester (EEUU) se validó la utilización de cogniciones o automensajes del tipo "Podemos hacer algo más que sufrir". Estos mensajes funcionaron como narrativas coherentes y esperanzadoras para la población. Así mismo, los niños soldados con bajas cogniciones de culpa y menor motivación de venganza (expuestos a la violencia en Uganda), tampoco mostraron sintomatología psiquiátrica (Klasen, 2010).

En la misma línea, el "Modelo de conceptualización de los supervivientes" del holocausto judío pone de manifiesto la existencia de factores de protección, como el transmitir valores y educar con memoria a la siguiente generación (Cohen, 2010). En una muestra de 133 supervivientes del holocausto judío, darle un sentido a los eventos, adaptarse y crecer funcionaron como factores de protección (Greene, 2010).

Igualmente ocurre con la muestra de mujeres víctimas de violencia sexual en Ruanda, que relacionaban su mejoría emocional con la neutralización del estigma al dar un significado al trauma (Zraly, 2010). Y en el estudio de los niños refugiados de Svensson (2009) dar significado al trauma a través del dialogo mejoro su sintomatología psiquiátrica.

La esperanza o "Sense of hope" también puede ser un potencial activador de recursos que aparece en la investigación. En el estudio sobre como las personas superan los riesgos en contextos de adversidad en Afganistán, en 2006 se realizaron entrevistas cara a cara a 1011 niños de 11 a 16 años y a 1011 adultos que les cuidaban. Para ambos grupos, la esperanza, como valor familiar y de unión fue el pilar sobre el que sostenerse ante las adversidades (Eggerman y Panter-Brick, 2010).

Por último, para dar sentido al trauma, el uso de las cogniciones, también fue valorado por los refugiados en Rorchester, NY. Este grupo afirma haber utilizado mensajes que diferenciban su identidad del hecho del trauma, como "We can do something more than suffer", o el uso de "Double consciousness," como pensar y no pensar en el trauma continuamente. Otras cogniciones motivacionales e ilusiones tras la vivencia traumática, como por ejemplo las ganas de emprender viajes, o sueños nunca realizados y la capacidad de recordar lo que les movió a irse, fueron otro de los factores que ayudaron a su adaptabilidad (Boucher, 2009).

\subsubsection{Factor B: Recursos y descripción de los factores de resiliencia:}

El Factor B, suponen los recursos que pongo en juego, tanto de los miembros individualmente, como de la familia y su red de apoyo social. Son los recursos existentes previos a la crisis, como los que la familia pone en marcha posteriormente y median entre la acumulación de demandas y la adaptación familiar.

La literatura sobre los factores de protección es amplia y sitúa estos factores en tres niveles: individual, familiar y social. Conviene aclarar como suelen 
funcionar en conjunto y como ninguno de ellos por sí mismo es determinante.

\section{A Nivel Individual:}

Edad: No existe un acuerdo sobre si las personas jóvenes se adaptan mejor que las mayores. En principio se podría pensar que se adaptan mejor, pero las crisis a edades muy tempranas también suponen riesgos para el desarrollo. Especialmente se han encontrado resultados donde la edad es un factor de riesgo para el afrontamiento del contexto de refugio, siendo los más jóvenes los más vulnerables (Wickrama y Kaspar, 2006).

Conocimiento de la lengua del país de destino: Los recursos a la llegada, como conocer la lengua del país, son fundamentales para la adaptación. En la muestra de refugiados asentados en Canadá, fueron las dificultades de comunicación, la dificultad del lenguaje y las diferencias culturales, las que generaron mayor estrés (Kirmayer, 2011).

Educación: la formación y educación de las personas refugiadas en Kakuma,(Kenia) así como, el nivel de capacidades humanas y psicológicas y el bagaje previo, fueron algunos de los factores protectores observados que más valoraban para su adaptación (Horn, 2009). En el estudio de los menores refugiados en Dinamarca, se analizan y comparan factores previos (número de traumas, intensidad antes de su llegada, la educación de la madre), y otros posteriores (experiencias de discriminación en el nuevo colegio, el cuidado de los profesores, mantener o no su vida religiosa, el género, o la integración cultural). Como conclusión se observa que la madre reciba educación en el país de destino es un factor de resiliencia (Montgomery, 2008).

Perspectiva positiva: Walsh (2004) define el enfoque positivo como la perseverancia, el aliento, el coraje, la esperanza enfrentar los hechos traumáticos. Así como el apoyo en puntos fuertes y la dominación de lo posible y la aceptación de lo que no se puede cambiar. Los refugiados en Rochester, NY, reconocen el uso de mensajes positivos en su cabeza favorecer su autoestima y sentimientos de bienestar (Boucher, 2009).

Transcendencia y espiritualidad: Las creencias espirituales o trascendentes son fuentes de significado, y de propósito que van más allá de nosotros y de nuestra adversidad. Los refugiados en Rochester, NY, ponen de relieve la importancia para ellos de la fe, de rezar cada día (Boucher, 2009). En la muestra de niños soldados de Uganda los factores de resiliencia se relacionaron con mayor soporte espiritual percibido (Klasen, 2010).

Humor: El humor, o la risa fue otro de los factores utilizados para superar la adversidad en el grupo de refugiados en Rochester (Boucher, 2009) .

Capacidad artística, creatividad: La capacidad de expresión artística puede constituir una forma de comunicación sanadora, cuando no se conoce el idioma supone una forma de no aislamiento de resolución del trauma. Los refugiados en Rochester comentaban entre las actividades creativas que les sirvieron de sostén: 
"cocinar, bailar, plantar, pintar" (Boucher, 2009).

Actitud pro-activa a la hora de integrarse: La actitud a la hora de integrarse, (King, 2010) ha sido estudiada en el estudio cualitativo de las familias refugiadas de Yugoslavia, resultado de la guerra de los Balcanes de 1990, en el sur de Australia. A través de entrevistas semi-estructuradas, para examinar la experiencia de antes, durante y después de los conflictos, se identifican 3 patrones: integración activa, pasiva y segregación a la hora de integrarse. Los resultados muestran la relación de la integración activa con la adaptabilidad familiar.

Otras capacidades emocionales: Se han encontrado evidencias de habilidades emocionales que funcionan como factores de resiliencia, como el autocuidado o la auto-regulación. En la muestra de refugiados asentados en Estados Unidos, el auto-cuidado de sus cuerpos, imagen, e higiene fue un factor de protección emocional ante la adversidad (Boucher, 2009). Así como en el estudio de Archuleta (2015) los adolescentes migrantes llegados a Estados Unidos, relacionaron su bienestar emocional y capacidad de auto-regulación emocional.

\section{A Nivel familiar:}

La resiliencia familiar ha estado muy debatida. Uno de los aspectos más llamativos de la definición de Manciaux (2003) es que incluye al "grupo", como agente resiliente, lo cual nos hace pensar las posibilidades de la familia como grupo, o agente de resiliencia, no solo a los menores afectados de forma individual. El enfoque familiar de la resiliencia, no ha sido común en los primeros años de desarrollo teórico. Se comenzó apostando por un enfoque individual, de factores que un miembro del sistema familiar desarrollaba a "a pesar" de la disfuncionalidad de la misma. Por lo tanto se ha conceptualizado la familia como carente de resiliencia, enfocándola como causante de patologías. Walsh (2004) propone una mirada desde sus capacidades, en vez de mirar individuos resilientes y familias perturbadoras. Son numerosos los estudios de investigación que evidencian que los factores de protección familiar guardan relación con el bienestar de los hijos. En el estudio piloto de 12 menores marroquíes no acompañados, mejora su sintomatología, al iniciar el trabajo de búsqueda de su red social y apoyo familiar e incorporarlos al programa. Se mide depresión, autoestima, alexitimia y relaciones familiares. Los resultados mostraron en el pre-test y post-test variabilidad con y sin familia. La sintomatología en las variables medidas disminuye al incluir en la segunda fase a la familia. Concluyen que trabajar con la familia en origen de los menores no acompañados es un factor relevante (Martinez de Apellániz Alday Ochoa, 2009). En las familias camboyanas refugiadas en EEUU, el mantener su identidad familiar y el contacto en ambos países, se convierte en un factor de protección. Un ejemplo de esto serían las llamadas "familias transnacionales" que mantienen el contacto con su familia en el país de origen y a su vez se integran en el país de destino (Lewis, 2010).

La memoria familiar: Numerosas investigaciones avalan la memoria como 
factor de resiliencia, y en concreto la colectiva y familiar. Las narrativas, la transmisión generacional, contar sus historias a otros y compartirlas a través de distintas formas como el método de "Collective narratives timelines", fue un recurso protector para la muestra de refugiados en Rochester. Así mismo, a la hora de afrontar el trauma recomiendan el "perdonar y recordar" a la vez (Boucher, 2009). A igual que para los supervivientes del holocausto judío, en el estudio que revisa 40 memorias, 60 años después de su liberación. Fueron elegidos al azar del estudio previo de John Templeton Fundation "Forgiveness, resiliency, and survivourship among holocaust survivours". Como factores de protección aparecen: la reconstrucción familiar de los hechos, el transmitir valores, contribuir a la comunidad y el educar a la siguiente generación (Cohen, 2010).

Figuras de apego con patología: Como ya hemos mencionado, la salud mental de las figuras de apego es un factor protector, que en su ausencia es un importante riesgo.

Parece que todavía está en cuestión, si los padres afectados por traumas son capaces de ser tutores de resiliencia de sus hijos o deben ser "sustituidos" por otras figuras como los profesionales. En el estudio del tsunami de Sir Lanka, las madres deprimidas correlacionaron con mayores síntomas de TEPT en sus hijos adolescentes. Parece que los menores son más vulnerables a la sintomatología parental, para sostenerse frente a la adversidad (Wickrama y Kaspar, 2006). En el estudio de Montgomery (2008) fue la importancia del vínculo con la madre y su salud mental es un factor protector de los menores refugiados en Dinamarca, en su proceso de asentamiento a su llegada. El objetivo del artículo fue aclarar la influencia de las experiencias traumáticas antes de la migración, frente al impacto de la vida social y redes a la hora de instalarse en el país de destino. La población fueron 131 niños y adolescentes (15,3 años de media) refugiados que llevaban entre 8 y 9 años asentados y 67 familias. Se estudiaron los efectos a largo plazo en estos menores, tanto a nivel individual, familiar como comunitario. El estado de salud mental de la madre, resultó ser relevante para la estabilidad de los hijos.

Vaage (2011) aporta una perspectiva longitudinal de niños y adolescentes refugiados vietnamitas $(\mathrm{N}=94)$ y sus familias asentados en Noruega durante 23 años, recogiendo la historia, exilio y adaptación. Con el objetivo de analizar la asociación entre estrés psicológico y la sintomatología familiar, en busca de predictores parentales posibles causantes de patologías en los hijos. El método utilizado fue la información recogida de los padres a su llegada en 1982 (T1), después de tres años en 1985 (T2) y 23 años después (T 3) de su llegada y asentamiento. Los instrumentos utilizados para medir salud mental fueron el "Global severity index GSI", SCL90 de síntomas de patología (ansiedad, depresión, agresividad...).Para los niños (4-18 años), usaron el Strenghts and difficulties questionnaire (SDQ). Los resultados muestran que del total de la muestra, el 30\% de los niños tenía un padre/madre con sintomatología psiquiátrica. Un predictor negativo de salud mental encontrado fue, que de los padres con TEPT en estado T3 
correlacionaban con hijos con sintomatología psiquiátrica. Se puede concluir que los hijos de refugiados no tienen por qué ser una población patológica forzosamente, pero si el padre tiene sintomatología de TEPT si hay que considerarlo como un factor de riesgo.

No se encuentran estudios que demuestren que los traumas en padres no generan traumas en hijos, pero si existen estudios realizados en población judía, que a través de la resignificación del trauma (narrativas familiares), parecen proteger a la siguiente generación. A este fenómeno se le ha llamado la "La transmisión del trauma entre generaciones" y se ha analizado en víctimas del Holocausto judío (Cohen, 2010) .

Mantener las raíces familiares, cultura, etnicidad, identidad familiar: El concepto de etnicidad también está poco consensuado. Falicov (2011), insiste en la importancia de mantener la cultura propia, la identidad y las tradiciones del país de origen, como factor de protección en los migrantes económicos. Se trata de mantener vivo un sentimiento positivo, de pertenencia a unas costumbres, lengua y raíces.

En el estudio de la comunidad de refugiados de Melbourne, Australia (CorreaVelez, Gifford y Barnett, 2010). Se entrevistan las familias con hijos de 11 a 19 años de edad, donde la identidad familiar se convierte en un factor de protección, junto con el sentimiento de pertenencia a su familia, a su comunidad y al sitio donde se han reasentado. Igualmente la preservación de los valores tradicionales de los Jemeres Rojos, pueden explicar la adaptación positiva a un país hostil (Rousseau \& Drapeau, 2003). Chrispin (2012) también analiza la importancia del apoyo familiar y mantener las raíces culturales del país de origen en una muestra de adolescentes Haitianos en América sometidos a altos niveles de estrés. Parece que la influencia de los padres o familia fue un protector para el bienestar emocional de los adolescentes. De la misma forma que los adolescentes migrantes del estudio de Carmeron, Pinto y Tapanya (2014) que aumentaron su autoconfianza, satisfacción con la vida y crecimiento personal, gracias a compartir actividades familiares y tener apoyo de sus padres.

Capacidad empática, de contención, comunicación y expresión emocional: La buena comunicación familiar es el engranaje de las relaciones, lo que favorecen la superación de situaciones traumáticas. Bajo este título Walsh (2004), incluye la claridad, la expresión emocional, y la resolución cooperativa de problemas. La claridad hace alusión a los mensajes claros y coherentes, la expresión emocional se refiere a compartir sentimientos, empatía, tolerancia, responsabilidad de las emociones de cada uno y el humor. Algunos de estos factores ya han sido mencionados en contextos de refugio, en las investigaciones de Boucher (2009) como el apoyo mutuo familiar y la expresión emocional.

Aceptación del ciclo vital: Las familias funcionales tienen un concepto del tiempo y del devenir, como un proceso continuo de crecimiento y cambio que progresa a lo largo del ciclo vital (Walsh, 2004). Algunas familias ante la adversidad 
y un contexto amenazante para ellos, se aglutinan, de forma que el ciclo se pueda estancar y con ello el crecimiento de los hijos. Es importante continuar con el ciclo vital familiar, a pesar de estar en el exilio, o con dificultades de adaptación. En el Campo de Kakuma (Kenia) uno de los factores de riesgo que aparecen, asociado a la desesperanza, fue el no tener un futuro sobre el que proyectarse (Horn, 2009). Los jóvenes refugiados sin posibilidad de trabajar, pueden desmotivarse sin posibilidad de proyectarse en el futuro y con sus etapas de desarrollo vital congeladas.

Rituales: Los rituales son otra de las formas que mantiene vivas las redes comunitarias y dan una identidad a las personas que participan de ellos. Falicov (2011), en su análisis de las familias inmigrantes económicos, o familias transnacionales insiste en la importancia de no perder tus raíces, o lo que llama "no lavar la tierra de las raíces de la planta que cambias de sitio", para conservar una estabilidad y salud familiar. Los rituales de paso, el contacto con las redes sociales étnicas, reproducir los espacios culturales propios en el nuevo país de destino mejoran la depresión en mujeres y los síntomas de agresividad en hombres. Boucher (2009) también menciona entre sus resultados, las tradiciones y las celebraciones de sus ritos como factor de protección.

Resolución de conflictos: La resolución de las crisis y problemas familiares desde la alianza entre los padres favorece al sistema familiar. Walsh (2004) se refiere a una resolución cooperativa con un liderazgo compartido de los padres, como "arquitectos de la familia". En los contextos de refugio las familias pueden ser variadas en su estructura, monoparentales, biparentales o con figuras de abuelos o tíos reasentados juntos. Para dar estabilidad a los hijos es importante la cooperación de los adultos y el apoyo mutuo, en la resolución de conflictos. Entre los refugiados asentados en Australia originarios del cuerno de África, se señalan, como uno de los obstáculos para la integración fueron los conflictos familiares (Shteir, 2009).

\section{A Nivel Social:}

Parece que la resiliencia es social, familiar, contextual y comunitaria. Todas estas palabras son sinónimos de red social y apoyo para reconstruirse. "No es posible reconstruirse solo", como señala Barudy (2006), por lo tanto la importancia de las figuras o tutores de resiliencia es vital. Así el contexto, los apoyos sociales, amigos o comunidad pueden funcionar como "tutores de resiliencia".

Apoyo comunitario: Las comunidades de origen de las personas desplazadas, funcionan como familias sustitutas en el contexto de refugio. El enfoque comunitario de resiliencia fue un recurso de protección espontanea del pueblo Tamil de la India, durante su asentamiento en Noruega (Guribye, 2011).Su mecanismo sanador, tuvo que ver con aprovechar los recursos comunitarios compartidos y darle un significado cultural en comunidad. Los imprevistos fueron tomados como "desafíos" y les obligaron a adaptarse y desarrollar más estrategias de superación comunitarias. Definen su experiencia como: "Desarrollo de estrategias comunales 
proactivas de superación, o el proceso por el cual un grupo se sienten responsables de su futuro bien estar y cooperan para promoverlo y prevenir indeseables cambios" (Guribye, 2011).

En el Campo de refugiados de Kakuma (Kenia) concluyen como una de las mayores pérdidas ocurridas durante el desplazamiento fueron: la hostilidad entre grupos sociales. Y añaden como factor de protección las redes sociales y la estructura comunitaria (Horn, 2009).

Así mismo, en la muestra de 136 migrantes mexicanas (Marsiglia, 2011)la diferencia encontrada entre las que desarrollan sintomatología depresiva y desesperanza, y las que no, fueron las que mantuvieron un sentido de pertenencia a su comunidad. Igual que ocurría con la muestra de mujeres víctimas de violencia sexual de Ruanda, que relacionaron su mejoría con estar conectadas a una red social (Zraly, 2010). Tras la experiencia del huracán Katrina, en un estudio cuatro años después (Bava, 2010) se ven como factores sanadores, la colaboración para resolver lo que ocurrido en comunidad, entenderlo juntos y la participación en actividades comunitarias.

Un resultado positivo encontrado en la muestra de padres refugiados en Noruega, fue que los que habían conseguido una mayor inserción y red social 23 años después, no encontraron patología en los hijos (Vaage, 2011) Los refugiados asentados en Rochester, también señalaron que las fortalezas comunitarias les ayudaron a sostenerse durante los efectos del trauma (Boucher, 2009). Este factor, en su ausencia se convierte en un riesgo, como analizan en la investigación sobre la exposición a la violencia en Palestina. Los resultados peores pronósticos de recuperación, cuando la acumulación de estresores unido a la falta de apoyo social (Hobfoll, 2011).

El contexto de llegada: La influencia del contexto de acogida es otro factor clave. En el estudio de Horn (2009) la sintomatología de los refugiados disminuye con forme tienen apoyo social y red comunitaria en el campo. Los participantes admiten que de los países con violencia de donde provenían, las condiciones eran más duros, pero tenían familia y red de apoyo social.

En cambio en los campos de refugiados a menudo, han sido separados o las redes de su comunidad y cultura son más débiles lo que aumenta sus sentimientos de desesperanza.

En una muestra de refugiados ( 19 a 40 años de edad) sudaneses que vivían en Australia desde hacía 5 años, realizan entrevistas y "freelisting" con lo que fueron para ellos "ayudas y estorbos" para su integración. Entre las conclusiones encuentran como, no solo influyen los traumas del pasado, sino también las dificultades de adaptación al nuevo país son importantes para la superación (Shakespeare-Finch, 2010).

Recursos materiales a la llegada: En el estudio de los refugiados en Afganistán, en 2006, para las personas entrevistadas, los aspectos que más les afectaban eran la pobreza económica, social y la falta de educación (Eggerman \& 
Panter-Brick, 2010).

Los refugiados asentados en Australia originarios del cuerno de África, se observan como los obstáculos para la integración fueron: la falta de información, soporte y falta de ayuda para tener una casa a su llegada (Shteir, 2009) .

Políticas de integración y apoyo: Una de las necesidades que favorecen una adecuada integración de los menores refugiados, son las sociales y emocionales a su llegada en el país de acogida. Las experiencias de los niños en el país de origen, no solo son de pérdidas, violencia o persecuciones empeoran, si a la llegada o durante la migración sufren potenciales traumas y tienen consecuencias sobre el desarrollo de niños y jóvenes aun en crecimiento (Hart, 2009).

\section{Conclusiones:}

Para terminar, se han elaborado las siguientes conclusiones y propuestas de futuras líneas de investigación:

1-Es necesaria una definición unificada de la resiliencia, y una perspectiva común para los futuros estudios. De forma que cuando se analice el constructo nos podamos referir al mismo, con sus cualidades y características comunes. Facilitaría el avance científico, las comparaciones entre distintas muestras y los análisis. Así mismo, en el ámbito de la intervención ayudaría al diagnóstico, prevención, tratamiento y evaluación de la resiliencia en las poblaciones afectadas por traumas.

2-También es necesaria una revisión de los métodos de medida ya existentes de la resiliencia, tanto para el avance científico como para la intervención. Sobre todo si la comprendemos como un constructo en interacción dentro de un contexto social y/o familiar.

3-En el contexto de refugio, parece que la resiliencia no se relaciona tanto con el tipo de trauma, su intensidad o características, o los factores de riesgo sino con la adaptación posterior o el contexto de "acogida" del trauma. Ahí se ponen en juego la resiliencia en todas sus formas. Una interesante línea de investigación sería la comparación de los campos de refugiados, como contexto de acogida y otros contextos de refugio donde se permita elaborar un proyecto de futuro, trabajo y demás factores protectores.

4-El tener factores protectores en distintas etapas del proceso de la migración, favorece la mejor planificación de la intervención terapéutica. Este es uno de las claves que se podrían tener en cuenta para la revisión de los métodos de intervención psicoterapéuticos con refugiados. Pudiendo incidir en distintos momentos del proceso, conociendo su eficacia.

5-Hay una falta de consenso general sobre qué factores son de protección y cuáles de riesgo para el desarrollo de la resiliencia. El género, la edad y la familia, son dos buenos ejemplos de estudios con resultados contradictorios.

6- Por último queda abierto el debate sobre la familia como su propio tutor de resiliencia en contextos de refugio. En las investigaciones encontradas destacan los estudios de figuras parentales con sintomatología como predictores negativos de 


\section{síntomas en los niños. Sería una interesa línea de investigación saber si existe el} efecto contrario.

\section{Referencias bibliográficas}

ACNUR (2014). Tendencias de asilo 2014. Niveles y tendencias en paises industrializados. Genova: ACNUR.

ACNUR. (2015). Tendencias globales. Desplazamiento forzado en 2015. Forzados a Huir. Recuperado de: http:/ /www.acnur.org/fileadmin/Documentos/Publicaciones/2016/10627.pdf?view=1.

ACNUR.(2016). Acnur. Recuperado el 03 de 10 de 2016, de ¿A quién ayudamos? Refugiados: http://www.acnur.es/ a-quien-ayudamos/refugiados

Asociación Americana de Psiquiatría, A. (2013). Manual Diagnóstico y Estadístico de los Trastornos Mentales. Washington.

Archuleta, A. (2015). Newcomers: the contibution of social and psychological well being on emotion regulation among firts generation acculturating latino youth in the southern United States. Child and adolescent social work journal, 32(2), 281-290.

Atwell, R. G.-W. (2009). Resettled Refugee Families and Their Children's Futures: Coherence, Hope and Support. Journal of Comparative Family Studies, 40(5), 677-697.

Bandura, A. (1977). Self-efficacy: toward unifying theory of behavior change. Psychological Review, 84, 191-215.

Barudy, J., \& Marquebreucq, A.-P. (2006). Hijas e hijos de madres resilientes. Traumas infantiles en situaciones extremas: violencia de género, guerra, genocidio, persecución y exilio. Madrid: Gedisa.

Bava, S. D. (2010). Lessons in Collaboration, Four Years Post-Katrina. Family Proces, 49(4), 543-558.

Bonnano, G. E. (2010). The paramenters of disaster: consequences, risks and resilience in individuals, families, communities, and society. Psychological Science in the Public Interest, 11, 1-49.

Boucher, M. (2009). Finding resiliency, standing tall: Exploring trauma, hardship, and healing with refugees. International Journal of Narrative Therapy \& Community Work, 4, 43-51.

Carmeron, C., Pinto, G., y Tapanya, S. (2014). Scaffolding one Thai youth's drawing toward resilience. Culture and psychology, 20(4), 453-476.

Chrispin, M. (2012). Resilient adaptation of church-affiliates young Haitian inmigrants: a search for protective resourses. International section A: Humanities and social sciences, 59(10-A), 3732.

Cohen, H. L. (2010). Memory and Resilience. Journal of Human Behavior in the Social Environment, $20(4), 525$.

Correa-Velez, I., Gifford, S., \& Barnett, A. (2010). Longing to belong: social inclusion and wellbeing among youth with refugee backgrounds in the first three years in Melbourne, Australia. Social science and medicine, 71, 1399-1408.

Delage, M. (2010). La resiliencia familiar. El nicho familiar y la superación de las heridas. Barcelona: Gedisa.

Eggerman, M., y Panter-Brick, C. (2010). Suffering, hope, and entrapment: Resilience and cultural values in Afganistan. Social science and medicine, 71, 71-83.

Falicov, C. (2011). Migración perdida ambigua y rituales. http://www.redsistemica.com, http:// www.redsistemica.com/ar/migracion2.htm.

Greene, R. R. (2010). Conceptualizing a Holocaust Survivorship Model. Journal of Human Behavior in the Social Environment, 20(4), 423-439.

Guribye, E. S. (2011). Communal proactive coping strategies among Tamil refugees in Norway: A case study in a naturalistic setting. International Journal of Mental Health Systems, 5(1),9-21.

Hart, R.(2009). Child refugees, trauma and education: interactionist considerations on social and emotional needs and development. Educational Psychology in Practice, 25(4), 351-368.

Hobfoll, S. E. (2011). The limits of resiliencie: Distresspolitical violence among Palestinians. Social science and medicine. Elservier, 72, 140.

Horn, R. (2009). A Study of the Emotional and Psychological Well-being of Refugees in Kakuma Refugee Camp, Kenya. Social Care, 5(4),20-32.

King, S. M. (2010). Serbian Stories of Translocation: Factors Influencing the Refugee Journey Arising from the Balkan Conflicts of the 1990s. Journal of Pacific Rim Psychology, 4(1), 61-71.

Kirmayer, L. J. (2011). Common mental health problems in immigrants and refugees: general approachin primary care. CMAJ: Canadian Medical Association Journal, 183(12), 959-967. 
Klasen, F. O. (2010). Posttraumatic Resilience in Former Ugandan Child Soldiers. Child Development, 81(4), 1096-1113.

Koch, S. C., y Weidinger-von der Recke, B. (2009). Traumatised refugees: an integrated dance and verbal therapy approach. Elservier. The art in psychotherapy, 36, 289-296.

Lewis, D. C. (2010). Cambodian Refugee Families in the United States: "Bending the Tree" to Fit the Environment. Journal of Intergenerational Relationships, 8(1), 5-20.

Manciaux, M. V. (2003). La resiliencia: resistir y rehacerse. Madrid: Gedisa.

Marsiglia, F. F.-P. (2011). Hopelessness, Family Stress, and Depression among Mexican-Heritage Mothers in the Southwest. Health \& Social Work, 36(1), 7-18.

Martinez de Apellániz Alday Ochoa, I. (2009). La importancia del trabajo con la familia en la atención a menores extranjeros no acompañados. Un estudio piloto. Apuntes de psicología, 27(2-3), 427-439.

McCubbin, H. y. (1983). The family stress process: the double ABCX model of adjustment and adaptation. Marriage and Family Review, 6, 7-37.

Montgomery, E. (2008). Long-term effects of organized violence on young Middle Eastern refugees'mental health. Social science and medicine, 67, 1596-1603.

Rosbrook, B. S. (2010). The meaning of home for Karen and Chin refugees from Burma: An interpretative phenomenological approach. European Journal of Psychotherapy \& Counselling, 12, 159-172.

Rutter, M. (1993). Resilience: Some conceptual considerations. Journal of Adolescent Health, 14(8), 626-631.

Shakespeare-Finch, J. W. (2010). Adaptation of Sudanese Refugees in an Australian Context: Investigating Helps and Hindrances. International Migration, 48(1), 23-46.

Shteir, S. P. (2009). "I Have a Voice-Hear Me!" Findings of an Australian Study Examining the Resettlement and Integration Experience of Refugees and Migrants from the Horn of Africa in Australia. Refuge, 26(2),133146.

Svensson, M.(2009). Making meaningful space for oneself: photo-based dialogue with siblings of refugee children with severe withdrawal symptoms. Children's Geographies, 7(2), 20.

Tomkiewicz, S. (2004). El surgimiento el concepto. En B. Cyrulniky otros, El realismo de la esperanza. Barcelona: Gedisa.

Torres Bernal, A. M. (2011). Healing from trauma: Utilizing effective assessment strategies to develop accessible and inclusive goals. KAIROS Slovenian Journal of Psychotherapy, 5(1/2), 28-42.

Vaage, A. B.-L. (2011). Paternal predictors of the mental health of children of Vietnamese refugees. Child $\&$ Adolescent Psychiatry \& Mental Health, 5(1), 1-11.

Walsh, F. (2004). Resiliencia Familiar.Estrategias para su fortalecimiento. Buenos Aires: Amorrortu.

Wickrama, K., \& Kaspar, V.(2006). Family context of mental health risk in Tsunami exposed adolescents: Findings from a pilot study in Sri Lanka. Social Science and medicine, 64(3),713-723.

Zraly, M. N. (2010). Don't let the suffering make you fade away: An ethnographic study of resilience among survivors of genocide-rape in south Rwanda.Social science and medicine, 70, 1656-1664. 